\title{
The linear separability effect in color visual search: Ruling out the additive color hypothesis
}

\author{
BEN BAUER, PIERRE JOLICOEUR, and WILLIAM B. COWAN \\ University of Waterloo, Waterloo, Ontario, Canada
}

\begin{abstract}
Bauer, Jolicoeur, and Cowan (1996b, 1996c) demonstrated difficult visual search for color targets that were not linearly separable (in color space) from two distractor colors and easier search for linearly separable targets. This suggested that search is mediated by a chromatically linear discrimination mechanism (see D'Zmura, 1991). However, in those experiments, the targets that were not linearly separable fell midway between the distractor colors and thus corresponded to the admix of the distractor colors. An alternate interpretation of the results of Bauer et al. is that search was more difficult when the target corresponded to the distractor admix than when it did not. We tested this hypothesis in three experiments by contrasting conditions in which a target that was not linearly separable did or did not correspond to the admix of the distractor colors. In all cases, a target that was not linearly separable produced difficult search, demonstrating that linear separability determines search performance.
\end{abstract}

D'Zmura (1991) and Bauer, Jolicoeur, and Cowan $(1996 \mathrm{~b}, 1996 \mathrm{c})$ reported that color visual search performance depended on the configuration of the target and distractor colors in CIE $(x, y)$ color space. When the target color fell midway between the distractor colors in color space, search times increased as the number of items in the search display increased. When the target color was off the line joining two distractor colors, or outside the triangle formed by three distractor colors, search slopes were much shallower. Bauer et al. (1996b, 1996c) proposed that if a search target can be segregated from the distractors in feature space (here color space) by a linear operator, search will be easy. If no such linear operator exists, search will be difficult. This property is sufficient to account for the results of dozens of visual search experiments reported by D'Zmura (1991) and Bauer et al. (1996b, 1996c). Schematically, this model can be depicted as in Figures 1 and 2. In both cases shown in Figure 1, the dashed line separates the target from the distractors in the color space shown. In these linearly separable cases, search slopes are shallow when compared with those that arise from the color configurations depicted in Figure 2. In Figure 2 , no single linear operator can simultaneously segregate the target from all the distractors. These configurations are not linearly separable, and search is difficult (Bauer et al., 1996b, 1996c).

This effect and the requisite conditions to elicit it are now well documented. The interpretation suggested by

This work was supported by the Natural Sciences and Engineering Research Council of Canada, and by the Human Frontiers in Science Program. The authors thank Jan Theeuwes, Frank Kooi, Jeremy Wolfe, and two anonymous reviewers for their helpful and constructive comments on earlier versions of this manuscript. Correspondence should be sent to P. Jolicoeur, Department of Psychology, University of Waterloo, Waterloo, ON, N2L 3G1 Canada (e-mail: pjolicoe@cgl.uwaterloo.ca).
D'Zmura (1991) and Bauer et al. (1996b, 1996c) is that search performance is mediated by a chromatically linear mechanism that permits easy detection of the target when the target color can be segregated from all the distractors by this mechanism. When the target cannot be detected by this mechanism, search is difficult. There is, however, another account that is consistent with the data reported by D'Zmura and Bauer et al. (1996b, 1996c). We call this account the additive color mixture hypothesis. When a target color falls midway between distractor colors, and when the colors are equiluminant, as was the case in D'Zmura and Bauer et al. (1996c), the target's chromaticity corresponds to the additive color mixture of the distractor chromaticities, provided that the distractors are present in equal numbers. Thus, the empirical result described by D'Zmura and Bauer et al. can be accounted for in two ways. According to the linear separability hypothesis, a target midway between distractors does not pop out because it is not linearly separable from the distractors. According to the additive color mixture hypothesis, a target midway between distractors does not pop out because it is equivalent to the average of the distractor chromaticities. The goal of the experiments reported in this article was to distinguish between these alternatives.

To determine which of the two hypotheses is correct requires a decoupling of two factors: linear separability and the correspondence of the average distractor chromaticity with the target chromaticity. Given the geometry of an equiluminant color plane, there are three ways that the target chromaticity and the additive color mixture of the two distractor chromaticities can be noncoincident. These three conditions are explained below.

If the target color is off the line joining the two distractors, the target will not be coincident with the additive color mixture of the distractors (see Figure 1, left panel). This was the case in D'Zmura (1991) and in Bauer et al. (1996b, 1996c) for their linearly separable conditions. Under 

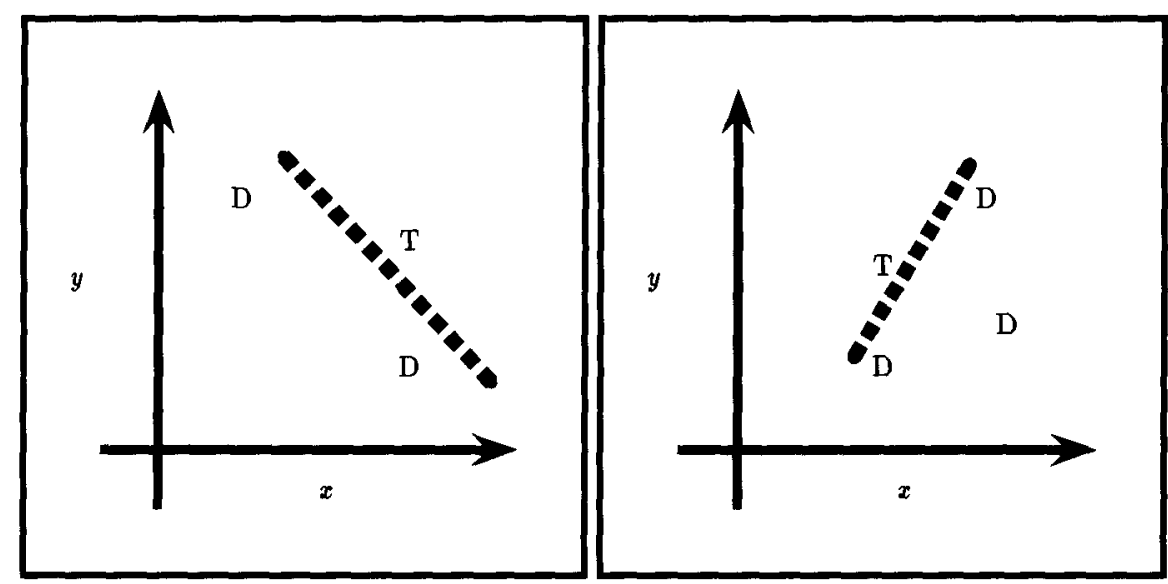

Figure 1. Linearly separable configurations (the linear operators are represented by the dashed lines). $T$ is the target, Ds are distractors (left, two distractors; right, three distractors). Search is relatively easy.

these conditions (when the target was off the distractordistractor line), search was easier than when the target was on the distractor-distractor line. In terms of the additive color mixture hypothesis, these results can be rephrased as follows: When the target chromaticity was coincident with the chromaticity of the additive mixture of the distractors, search was difficult. When the target chromaticity was not coincident with the additive color mixture of the distractors, search was easy. A condition in which the target does not fall between the distractors does not inform on the determination of which hypothesis is correct because both make the equivalent prediction of easy search.

A target can be directly between the distractor colors and nevertheless be different from the additive color mixture of the distractors. This can be realized in two ways. One way is to fix the distractors and move the target that is not linearly separable away from the midpoint position that corresponds to the admix of the distractor colors. The other way is to fix the target, but present the distrac- tors in unequal quantities so that the color corresponding to the admix of the distractor colors is pulled toward the more numerous distractor (away from the target). Both of these methods are developed more formally in the following paragraphs.

In the first method, the target chromaticity can be chosen such that it is closer to one of the distractors than to the other, with the distractors present in equal representation. This strategy holds linear separability constant because a target that is directly between the two distractors is not linearly separable regardless of its position relative to the distractors. To the extent that a target falls closer to one of the distractors (e.g., at $3 / 4$ or $1 / 4$ points between the distractors), it will no longer correspond to the admix of the distractor colors and remain not linearly separable. Using this method, the additive color hypothesis was put to the test in Experiment 3.

In Experiments 1 and 2, we used the second strategy, which involves a target midway between the distractors, but with the distractors presented in unbalanced quanti-

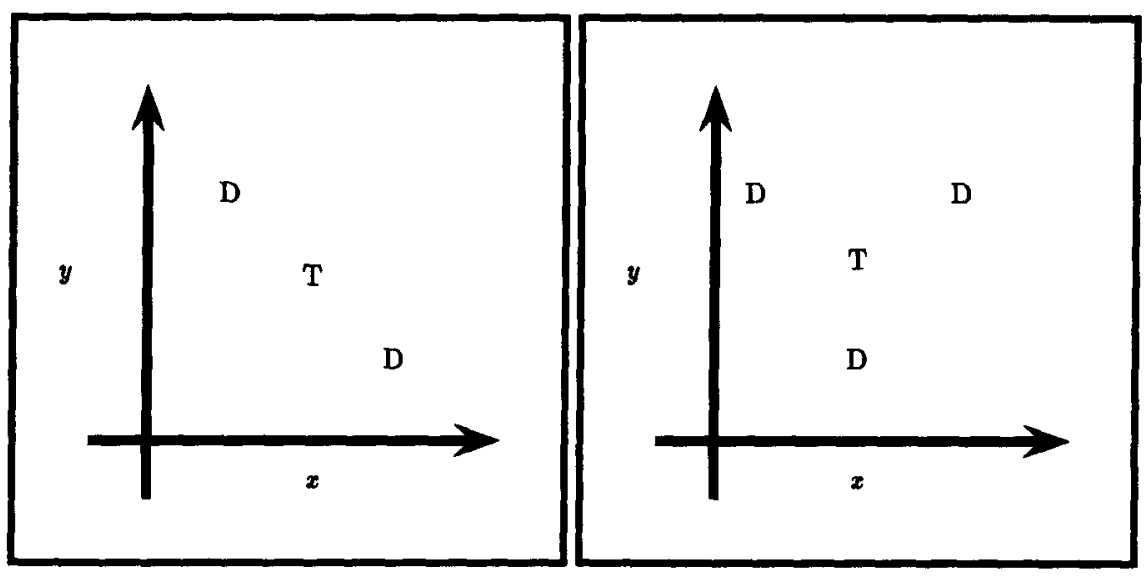

Figure 2. Configurations that are not linearly separable. $T$ is the target, Ds are distractors (left, two distractors; right, three distractors). Search is difficult. 
ties. In this way, we fixed the target position but the position of average of the distractor colors could be altered as a function of the ratio of the distractor quantities. If there are equal numbers of each distractor, the admixture is the simple average of the distractor chromaticities. If the ratio is not $1: 1$, the additive color is the weighted average (by quantity) of the distractor chromaticities. We used the ratios $1: 0,3: 1$, and $1: 1$. In the $1: 0$ case, the search is for a target in a homogeneous background of one or the other distractor. The $1: 1$ case is equivalent to that studied in D'Zmura (1991) and Bauer et al. (1996b, 1996c). In the $3: 1$ case, the global additive color mixture has coordinates that are pulled toward the overrepresented color. Specifically, if Distractor D1 has coordinates $\left(x_{1}, y_{1}\right)$ and Distractor D2 has coordinates $\left(x_{2}, y_{2}\right)$, and there are three times as many D1s as D2s, then the coordinates of the additive color mixture are

$$
\left(\frac{3 x_{1}+x_{2}}{4}, \frac{3 y_{1}+y_{2}}{4}\right) \text {. }
$$

In terms of search performance, the 1:0 case should produce very shallow slopes, indicating that, pairwise, the target is easily discriminable from either distractor. The 1:1 case should yield effortful search (and steep slopes). The slopes from the 3:1 condition relative to the other two conditions provide the critical test of the additive color hypothesis. Suppose that the slopes in the $3: 1$ condition are substantially lower than those in the $1: 1$ case, perhaps near those in the 1:0 case. This would suggest that the positioning of the target at the additive color point, rather than linear separability had been responsible for the effects reported in D'Zmura (1991) and Bauer et al. (1996b, 1996c). On the other hand, if the slopes in the $3: 1$ and $1: 1$ cases are similar, then it would be clear that the additive color hypothesis does not explain the effect. This last claim requires that the color whose coordinates are the $1: 1 \mathrm{mix}$ of the distractor colors be perceptually different from the color whose coordinates are the 3:1 mix of the distractor colors. Otherwise, it could be argued that, while the colors are different algebraically, they were not sufficiently different perceptually. This issue is addressed empirically by having the subjects search for the color whose coordinates correspond to the $1: 1 \mathrm{mix}$ in a field of distractors whose coordinates correspond with those of the $3: 1 \mathrm{mix}$. Easy search in this condition shows that the mean distractor color of the $3: 1$ mixture is sufficiently different from the target color to allow pop-out.

Using these two methods, we demonstrated in three experiments that the correspondence of the target with the admix of the distractors does not account for linear separability effects demonstrated by Bauer, Jolicoeur, and Cowan (1996a, 1996c).

\section{EXPERIMENT 1}

\section{Method}

Subjects. One female subject (T.O.) and 2 male subjects (B.B. [first author] and B.G.) were experienced psychophysical subjects, each having performed at least 10,000 visual search trials prior to this experiment. All had normal color vision as verified by normal performance on the Farnsworth-Munsell 100 Hues Test and on the Nagel anomaloscope. ${ }^{1}$

Equipment. Data were collected using an Apple Macintosh II computer with a RasterOps video board ( 8 bits per color gun) and an Apple high-resolution 13-in. RGB monitor ( $640 \times 480$ pixels). $\mathrm{CIE}(x, y)$ coordinates and luminance values (in $\left.\mathrm{cd} / \mathrm{m}^{2}\right)$ were measured using a Minolta Chroma Meter CS-100.

Stimuli. Stimuli were colored disks presented on a neutral background $(x=.287, y=.292$ ), which was equiluminous with the targets and distractors at $24.6 \mathrm{~cd} / \mathrm{m}^{2}$. Details regarding the measurement of the colors can be found in Bauer et al. (1996c). The colored target and distractor disks had a diameter of $0.76^{\circ}$ of visual angle. The colored disks were presented at locations on imaginary $6 \times 6$ grid with small, locational offsets ( \pm 3 pixels on $x$ and $y$, random on each trial, for each disk). The target never appeared in any of the four corner positions of the imaginary grid; within a block of trials, the target appeared equally often in the remaining 32 positions. The search array subtended about $8^{\circ} \times 8^{\circ}$ visual angle.

The coordinates of the colors are presented in Figure 3. The colors are plotted in CIE 1976 UCS, an ideally uniform color space whose metric $\left(\Delta E^{*}\right)$ is based on discriminability. Colors separated by equal distances $\left(\Delta E^{*}\right)$ will be roughly equally discriminable (CIE, 1978; Wyszecki \& Stiles, 1982).

For each of the 3 subjects, a different set of colors was used (indicated by the subjects' initials above each color line in Figure 3 ). For Subject B.B. (solid line) and for Subject B.G. (dotted line), the two farthest colors ( $\mathrm{D} 1$ and $\mathrm{Dl}^{\prime}$ ) were about $63 \Delta E^{*}$ units apart, with the colors of every nearest pair (i.e., D1-D2, D2-T, T-D2', $\mathrm{D} 2^{\prime}-\mathrm{Dl}^{\prime}$ ) about $15.5 \Delta E^{*}$ units apart. For Subject T.O. (dashed line), a well-practiced subject who had performed at least 35,000 visual search trials prior to the experiments reported here, D1 and $\mathrm{Dl}^{\prime}$ were about $53 \Delta E^{*}$ units apart, with colors of every nearest pair about $13 \Delta E^{*}$ units apart.

In the 1:0 ratio condition, the subjects sought the target $(\mathrm{T})$ in homogeneous fields of either of $\mathrm{D} 1$ or $\mathrm{D}^{\prime}$. In the $1: 1$ ratio condition, $T$ was the target, and there were approximately equal numbers of $\mathrm{Dl}$ and $\mathrm{Dl}^{\prime}$. In the 3:1 condition, the distractors were present in approximately $3: 1$ ratio. In a control condition, $T$ was sought in homogeneous backgrounds of colors D2 or D2'. D2 and D2' are at the weighted average chromaticity of D1 and D1' when these colors are present in a 3:1 or a 1:3 ratio mix. This control condition was designed to demonstrate that the target could indeed be discriminated from the colors corresponding to the $3: 1$ or 1:3 mixtures. In this twocolor control condition, the set sizes were 4,16 , and 36 , with set size, target position, and target presence or absence randomized within blocks of 192 trials.

In the ratio conditions, the target was color $\mathrm{T}$ and the distractors were the colors D1 and D1' (see Figure 3). These distractors were represented in the ratios of $1: 0$ and $0: 1,3: 1$ and $1: 3$, and $1: 1$ in set sizes of 16 and 32 as described in Table 1. Each block of 768 trials contained six logical types of trials in equal numbers: D1:D1'::1:0, $\mathrm{D} 1: \mathrm{D} 1^{\prime}:: 3: 1, \mathrm{D} 1: \mathrm{Dl}^{\prime}:: 1: 1, \mathrm{Dl}^{\prime}: \mathrm{D} 1:: 1: 0, \mathrm{D}^{\prime}: \mathrm{D} 1:: 3: 1, \mathrm{D} 1^{\prime}: \mathrm{D} 1:: 1: 1$. Trial types D1:D1'::1:1 and D1':D1::1:1 are identical trials as far as the subjects are concerned. To generate the trials, the distractor colors were allotted to the nontarget locations such that they were represented in approximate ratios of $1: 0,3: 1$, and $1: 1$. Table 1 illustrates the various quantities of the distractors for target-present and targetabsent trials across the set sizes of 16 and 32 items required to obtain the three different ratios. ${ }^{2}$ Note that, over a block of 768 trials, the ratios applied to both distractors. For example, for the set size 16 , target-present, 1:0 ratio condition, Distractor Dl would be absent and Distractor D2 would number 15, and on an equal number of trials, D2 would be absent and D1 would number 15 . The subjects completed two blocks of 768 trials. ${ }^{3}$ Within each block, trials of each ratio, target status (present/absent), and set size were intermixed. 


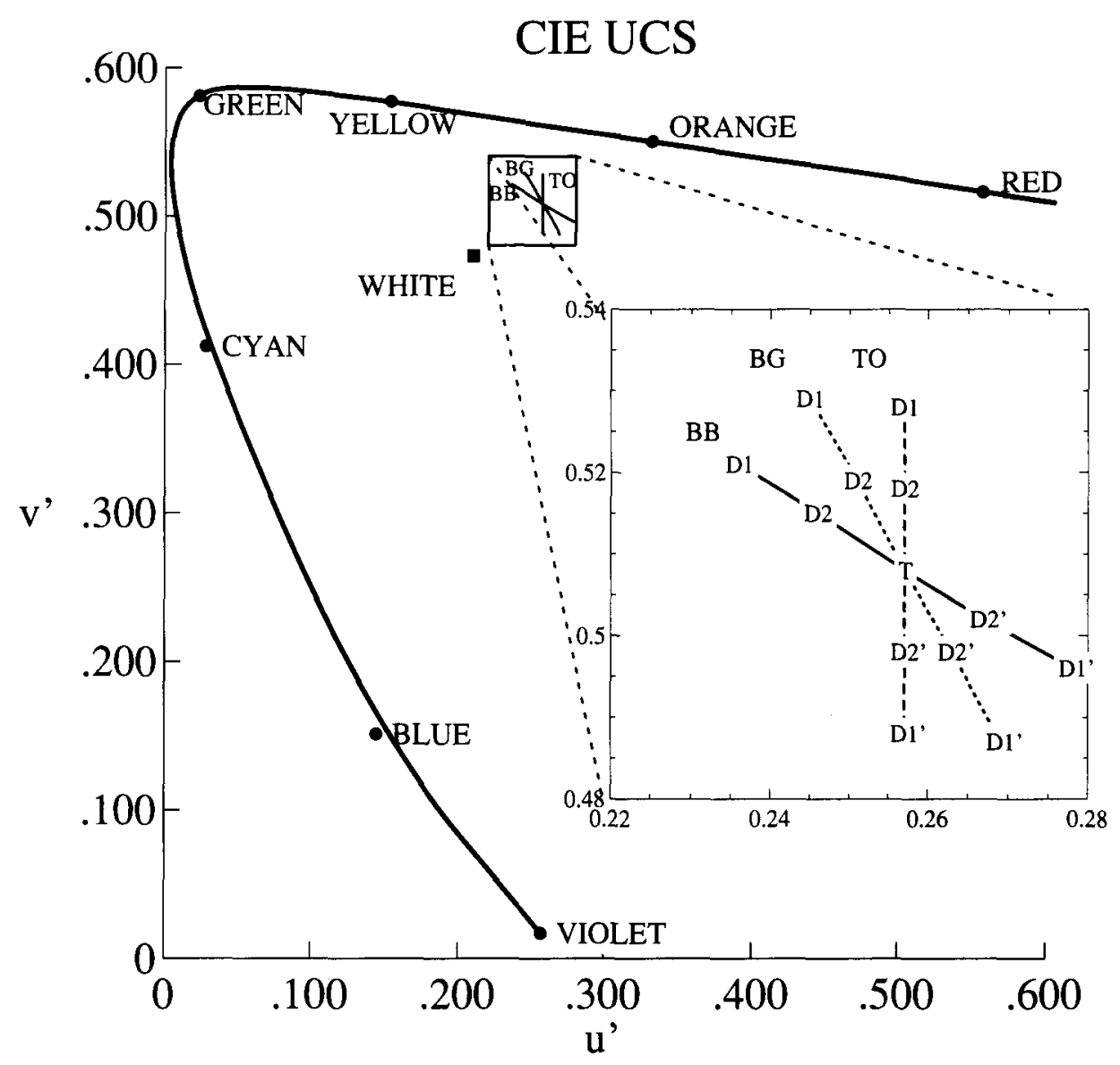

Figure 3. CIE 1976 UCS representation of colors. Enlarged area shows the configurations in more detail. The Appendix contains the CIE $x, y$ and CIE $u^{\prime}, v^{\prime}$ coordinates for these colors.

Procedure. Prior to each block of trials, there was a display with three colored disks indicating the target and distractor colors. The target-colored disk was presented just above the center of the screen with the word TARGET just above it. Two disks portraying the distractor colors were presented side by side just below the center of the screen with the word DISTRACTOR(s) just below. When the subject felt that he or she could remember the colors, a keystroke was given to start the trials. Forty practice trials preceded each block of 768 recorded trials. The subjects signaled the presence or absence of the target color by pressing the $\mathrm{M}$ or $\mathrm{C}$ key, respectively, on the Macintosh keyboard. Trials proceeded as follows: A "+" or a "-" was presented centrally for $400 \mathrm{msec}$ to signal a correct or an incorrect response on the previous trial (the first trial of a session was preceded by a "+"). The fixation symbol disappeared and was replaced immediately by the search display, which was terminated following a response. There was a $400 \mathrm{msec}$ pause before presentation of the next fixation. At the end of the practice trials, the number of correct responses out of 40 was reported to the subject. The recorded trials followed. There was a subject-timed break every 70 trials. At all times, the screen background was maintained at $24.6 \mathrm{~cd} / \mathrm{m}^{2}$, with CIE coordinates of $x=.287, y=.292$.

The procedure in the two-color control condition was similar except that there was only one target color and one distractor color per search, with 384 trials of T in D2 and 384 trials of T in D2'. Set sizes were 4,16 , and 36 .

Analysis. The reaction times (RTs) from "correct" trials were screened for outliers using the modified recursive outlier procedure with moving criterion described in Van Selst and Jolicoeur (1994). Reported RT slopes are based on linear fits to valid responses only-that is, RTs from "correct" trials that also passed the outlier procedure. As mentioned above, in the ratio conditions, there were equal numbers of trials in which Distractor Dl outnumbered Dl' as there were in which D1' outnumbered D1. Examination of the data including this factor revealed no consistent pattern. Data were collapsed over this factor. In the control condition, data were collapsed over the $\mathrm{T}$ in $\mathrm{D} 2$ and over the $\mathrm{T}$ in $\mathrm{D} 2{ }^{\prime}$ cells.

\section{Results}

The outlier elimination procedure resulted in the exclusion of $1.2 \%$ of B.B.'s RTs, $2.4 \%$ of B.G.'s RTs, and $2.2 \%$ of T.O.'s RTs. Individual subject's results are shown

Table 1

Experiment 1: Numbers of Distractors Used for Ratios 1:0, 3:1, and 1:1

\begin{tabular}{ccccc}
\hline & & \multicolumn{3}{c}{ Ratio } \\
\cline { 3 - 5 } Set Size & Target & $1: 0$ & $3: 1$ & $1: 1$ \\
\hline 16 & present & $15: 0$ & $12: 3 / 11: 4$ & $7: 8$ \\
16 & absent & $16: 0$ & $12: 4$ & $8: 8$ \\
32 & present & $31: 0$ & $24: 7 / 23: 8$ & $15: 16$ \\
32 & absent & $32: 0$ & $24: 8$ & $16: 16$ \\
\hline
\end{tabular}




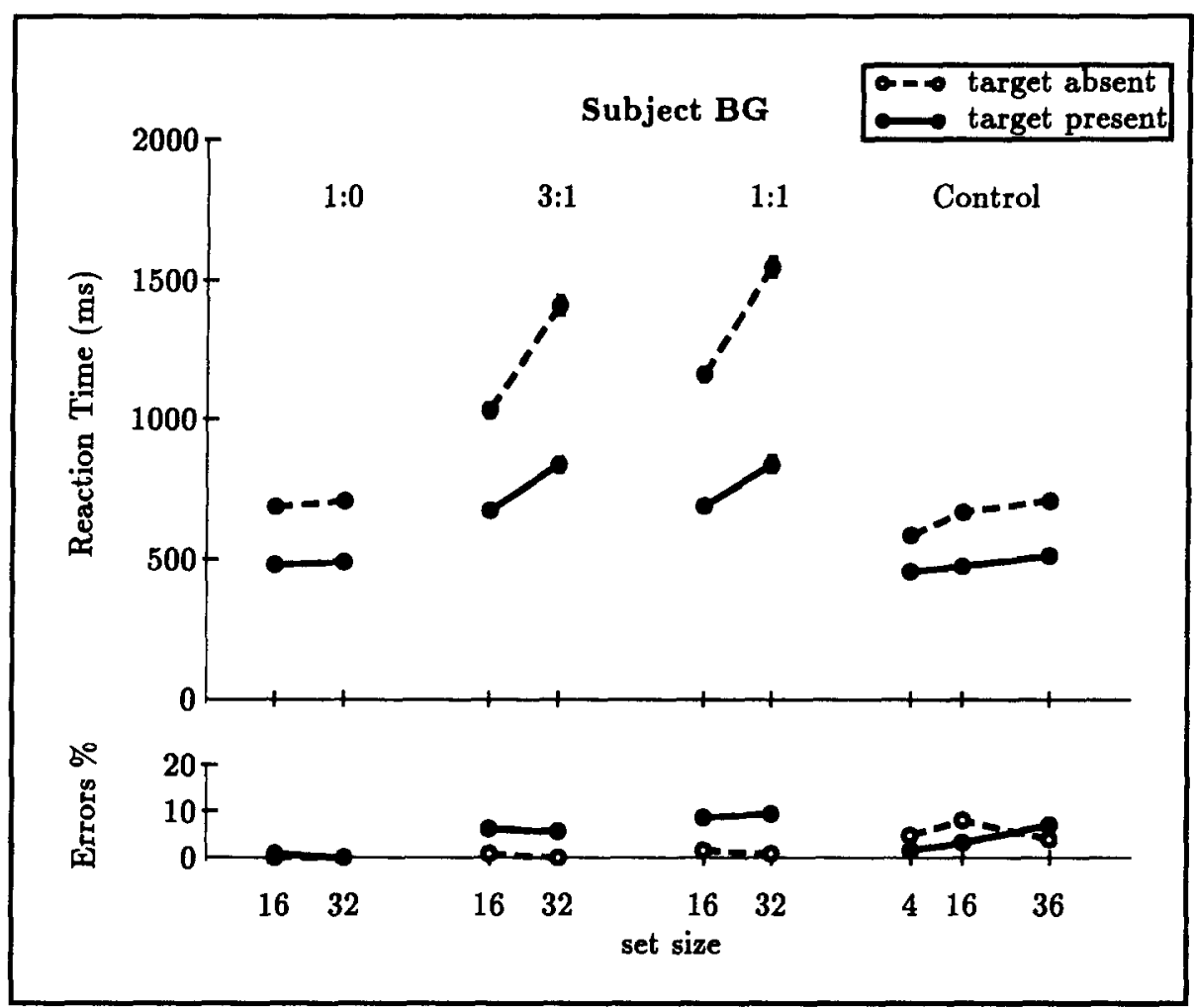

Subject BG: search slopes and error rates.

\begin{tabular}{|c|c|c|c|c|}
\hline \multirow[b]{2}{*}{ Condition } & \multicolumn{2}{|c|}{ Slope } & \multicolumn{2}{|c|}{ Mean \%Errors } \\
\hline & absent & present & absent & present \\
\hline $\begin{array}{c}\text { Control } \\
1: 0 \\
3: 1 \\
1: 1\end{array}$ & $\begin{array}{c}3.7 \\
1.2 \\
23.7 \\
24.0\end{array}$ & $\begin{array}{c}1.8 \\
0.6 \\
10.2 \\
9.3\end{array}$ & $\begin{array}{l}\mathbf{5 . 5} \\
0.0 \\
0.4 \\
1.2\end{array}$ & $\begin{array}{l}\mathbf{3 . 9} \\
\mathbf{0 . 4} \\
\mathbf{5 . 8} \\
\mathbf{9 . 0}\end{array}$ \\
\hline
\end{tabular}

Figure 4. Search results for Subject B.G. in Experiment 1. Reaction times (in milliseconds) and error rates are plotted in the upper panel. Search slopes (milliseconds/item) are presented in the table. Error bars $( \pm 1 S E M)$ may be smaller than the plotting symbol.

in Figures 4, 5, and 6. These figures show RTs and errors plotted in the upper panels, and corresponding slopes are tabled in the bottom panels.

The pattern of results can be summarized as follows: The slopes in the homogeneous control condition and those in the 1:0 ratio condition were small. This shows that $\mathrm{T}$ was easily discriminable from $\mathrm{D} 2$ and $\mathrm{D} 22^{\prime}$; $\mathrm{T}$ was easily discriminable from the color that corresponded to the additive mixture of $\mathrm{Dl}$ and $\mathrm{D} 1^{\prime}$ in a 3:1 proportion. Furthermore, $T$ was easy to find in homogeneous backgrounds of D1 or D1'. Of particular interest is the comparison between the $3: 1$ and $1: 1$ ratio conditions. There was scarcely any difference between these two conditions. Certainly, the additive color hypothesis would have predicted a blatant slope reduction in the $3: 1$ condition, and this was not manifest.
At a closer level of analysis, there appears to have been a slight RT advantage for the 1:1 condition on targetpresent trials; however, in 2 of the subjects, more errors were also made in the $1: 1$ condition, which could be interpreted as reflecting a speed-accuracy tradeoff. It is sufficient to say that the $3: 1$ and $1: 1$ ratio conditions re-

Table 2

Experiment 1: Group Average Search Slopes (Milliseconds/Item)

\begin{tabular}{cccccc} 
& \multicolumn{2}{c}{ Slope } & & \multicolumn{2}{c}{ Mean \% Errors } \\
\cline { 2 - 3 } \cline { 5 - 6 } Condition & Absent & Present & & Absent & Present \\
\hline Control & 4.8 & 2.5 & & 4.0 & 4.7 \\
$1: 0$ & 1.3 & 0.2 & & 0.3 & 0.3 \\
$3: 1$ & 19.6 & 10.6 & 1.2 & 8.3 \\
$1: 1$ & 20.3 & 9.7 & 2.4 & 9.1 \\
\hline
\end{tabular}




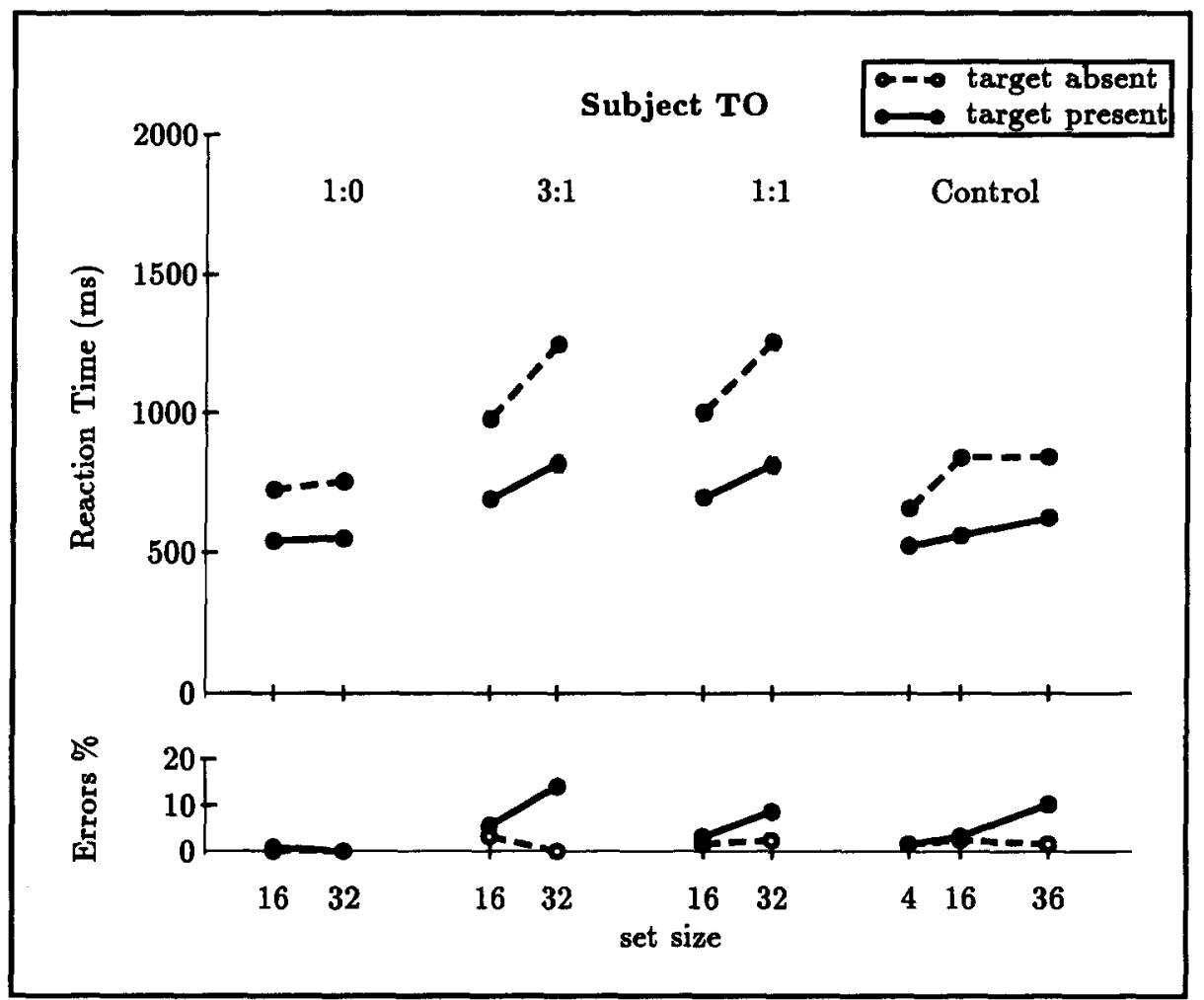

Subject TO: search slopes and error rates.

\begin{tabular}{|c|c|c|c|c|}
\hline \multirow[b]{2}{*}{ Condition } & \multicolumn{2}{|c|}{ Slope } & \multicolumn{2}{|c|}{ Mean \%Errors } \\
\hline & absent & present & absent & present \\
\hline $\begin{array}{c}\text { Control } \\
1: 0 \\
3: 1 \\
1: 1\end{array}$ & $\begin{array}{c}5.3 \\
1.8 \\
16.7 \\
15.7\end{array}$ & $\begin{array}{l}\mathbf{3 . 1} \\
0.8 \\
8.0 \\
7.2\end{array}$ & $\begin{array}{l}1.8 \\
0.0 \\
1.6 \\
2.0\end{array}$ & $\begin{array}{l}4.9 \\
0.4 \\
9.7 \\
5.9\end{array}$ \\
\hline
\end{tabular}

Figure 5. Search results for Subject T.O. in Experiment 1. Reaction times (in milliseconds) and error rates are plotted in the upper panel. Search slopes (milliseconds/item) are presented in the table. Error bars ( $\pm 1 S E M$ ) may be smaller than the plotting symbol.

sulted in very similar slopes, counter to the prediction made by the additive color hypothesis. As a summary of the overall pattern, the RT data for the 3 subjects in the ratio conditions were averaged and linear slopes fitted. These results are presented in Table 2 . The combined results reinforce the interpretations made above.

\section{EXPERIMENT 2}

One might be concerned that the $3: 1$ and $1: 1$ conditions produced such highly similar results because these conditions were mixed within blocks. To verify that the lack of a difference between these two conditions was not an artifact of the presentation method, we conducted Experiment 2 with ratios of $1: 0,3: 1$, and $1: 1$ presented in blocked fashion.

\section{Method}

Subjects. The subjects were B.B. and 2 new volunteers (S.R. [male] and E.O. [female]) who had performed several thousand trials in other color visual search experiments. S.R. and E.O. exhibited no color vision anomalies on the Farnsworth-Munsell 100 Hues Test and on the Nagel anomaloscope.

Equipment. Stimuli were presented using an Apple Power Macintosh 6100 and an Apple Multiple Scan 15-in. display. CIE $(x, y)$ coordinates and luminance values (in $\mathrm{cd} / \mathrm{m}^{2}$ ) were measured using a Minolta Chroma Meter CS-100.

Stimuli. The stimulus parameters (size, spacing, etc.) were the same as in Experiment 1 except that the background color was $x=$ $.300, y=.300$, and the luminance was $25.2 \mathrm{~cd} / \mathrm{m}^{2}$. For all 3 subjects, the target $\mathrm{T}$ had chromaticity $\left(u^{\prime}=.208, v^{\prime}=.543\right)$, and the distractors were D3 $\left(u^{\prime}=.228, v^{\prime}=.539\right)$ and D4 $\left(u^{\prime}=.187, v^{\prime}=.547\right)$.

Procedure. Each subject performed two sets of three blocks of trials. As in Experiment 1, these six blocks were of the trial types (D3:D4): 1:0, 3:1, 1:1, 0:1, 1:3, 1:1. For all trials, the set size was 


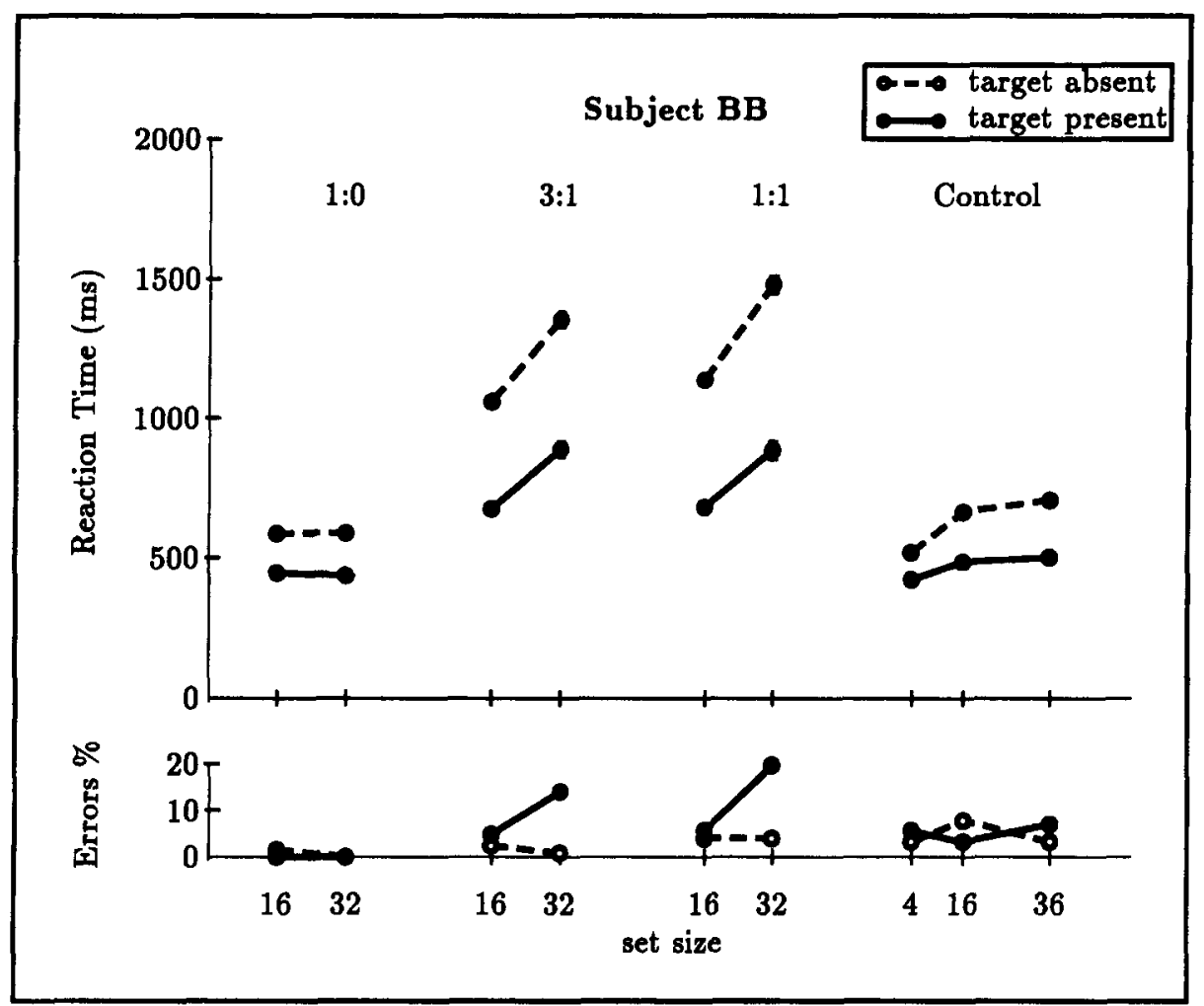

Subject BB: search slopes and error rates.

\begin{tabular}{cccccc}
\hline & \multicolumn{2}{c}{ Slope } & & \multicolumn{2}{c}{ Mean \%Errors } \\
\cline { 2 - 3 } \cline { 5 - 5 } Condition & absent & present & absent & present \\
\hline Control & $\mathbf{5 . 6}$ & $\mathbf{2 . 3}$ & $\mathbf{4 . 7}$ & $\mathbf{5 . 2}$ \\
$\mathbf{1 : 0}$ & $\mathbf{0 . 2}$ & -.5 & $\mathbf{0 . 8}$ & $\mathbf{0 . 0}$ \\
$\mathbf{3 : 1}$ & $\mathbf{1 8 . 5}$ & $\mathbf{1 3 . 4}$ & $\mathbf{1 . 5}$ & $\mathbf{9 . 3}$ \\
$\mathbf{1 : 1}$ & $\mathbf{2 1 . 3}$ & $\mathbf{1 2 . 6}$ & $\mathbf{3 . 9}$ & $\mathbf{1 2 . 5}$ \\
\hline
\end{tabular}

Figure 6. Search results for Subject B.B. in Experiment 1. Reaction times (in milliseconds) and error rates are plotted in the upper panel. Search slopes (milliseconds/item) are presented in the table. Error bars $( \pm 1 S E M)$ may be smaller than the plotting symbol.

fixed at 32 . This means that, for $1: 0$ and $0: 1$ trials, there were 32 of the given distractor on target-absent trials, and one distractor was replaced with a target on target-present trials. For $3: 1$ and $1: 3$ trials, there were 8 of one distractor and either 23 or 24 of the other distractor depending on target absence or presence. For $1: 1$ trials, the target replaced either a D3 or a D4 randomly. Each block contained 40 practice trials and 128 recorded trials. Trial presentation parameters (timing, feedback, etc.) were essentially the same as in Experiment 1.

Analysis. The data were first collapsed over like trial types to create three conditions. The 1:0 and $0: 1$ conditions were collapsed to create a condition we will refer to generically as $1: 0$. Similarly, $3: 1$ and $1: 3$ conditions were collapsed to create the $3: 1$ condition. Data from the two $1: 1$ blocks were aggregated into the $1: 1$ condition.

\section{Results}

The outlier elimination procedure resulted in the exclusion of less than $1 \%$ of B.B.'s RTs, $2.2 \%$ of S.R.'s RTs, and $1.5 \%$ of E.O.'s RTs. Individual subject's results are shown in Figure 7.

The 3:1 and $1: 1$ conditions provide the critical comparison vis-à-vis the additive color mixture hypothesis. If a target is hard to detect because it is equivalent to the average of the distractor chromaticities, then search in the $1: 1$ condition ( $T$ is the average of D3 and D4) should be difficult, whereas search in the $3: 1$ condition ( $T$ is not the average of D3 and D4) should be much easier.

\section{Discussion}

Inspection of Figure 7 reveals that performance was no better in the $3: 1$ condition than in the $1: 1$ condition. If anything, there appears to have been a small advantage for the $1: 1$ conditions. The group RTs are presented 


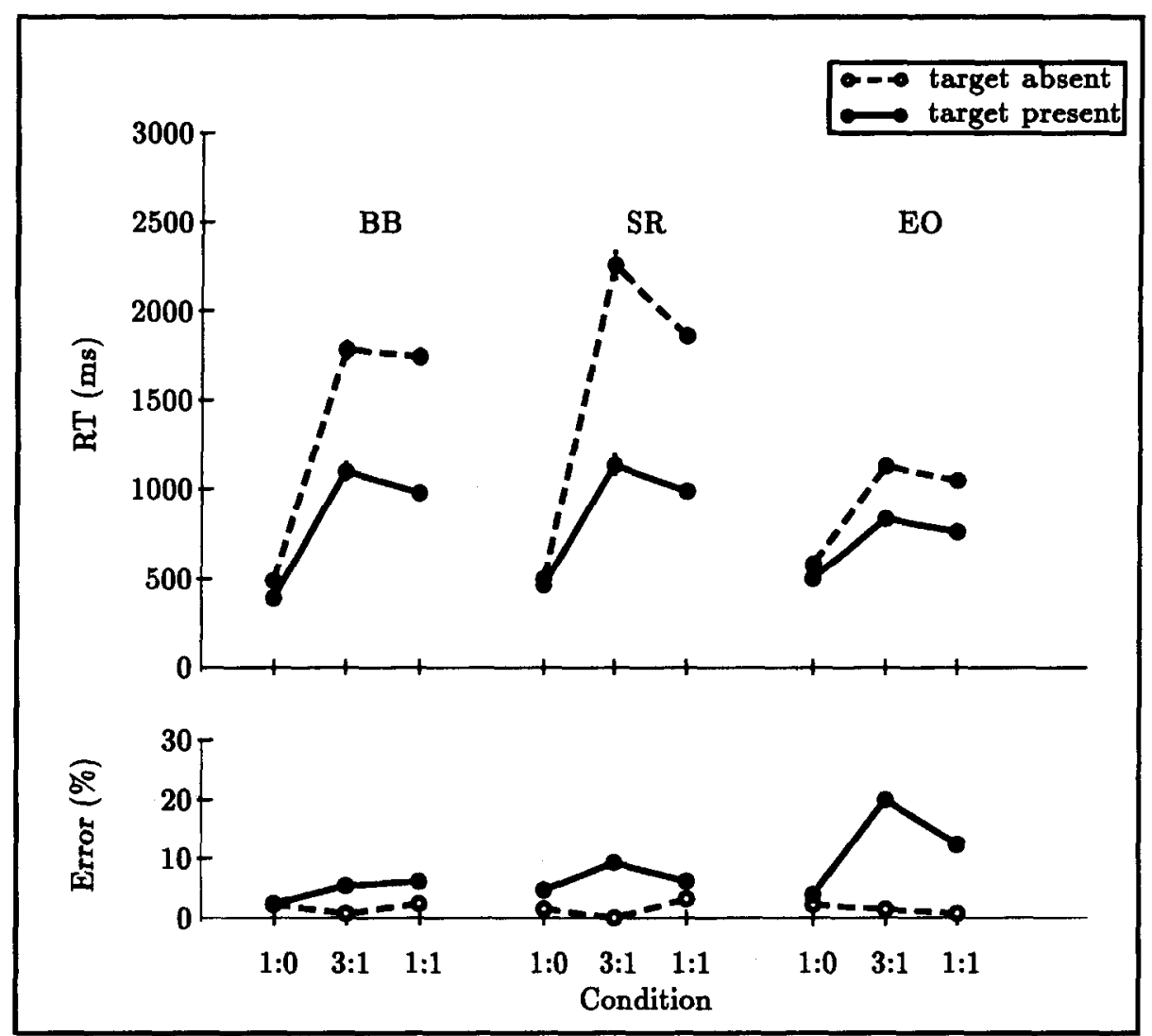

Figure 7. Search results for 3 subjects in Experiment 2. Error bars $( \pm 1 S E M)$ may be smaller than the plotting symbol.

in Table 3. We note that the lack of performance improvement in going from a $1: 1$ to a $3: 1$ ratio found in Experiment 1 (mixed presentation) and in Experiment 2 (blocked presentation) replicates a similar finding in Bauer et al. (1996a).

\section{EXPERIMENT 3}

In Experiments 1 and 2, we used a distractor-ratio manipulation to decouple linear separability and coincidence of the target with the additive mixture of the distractors. We found that search was no easier when the weighted average of the distractor chromaticities did not correspond ( $3: 1$ condition) to the target chromaticity than when it did (1:1 condition). This provides evidence that the linear separability effects reported in Bauer et al. (1996a, 1996c) were not a result of placing the chromaticity of the target that was not linearly separable at the point corresponding to the average of the distractor chromaticities. In Experiment 3, we provide one final experiment as converging evidence. Rather than using a distractor-ratio manipulation, we adopted the direct strategy of selecting three different target chromaticities that were not linearly separable from a pair of distractors (i.e., that fell on an imaginary line joining the two distractors in color space). One target corresponded to the average of the two distractors. This target should produce difficult search as in Experiments 1 and 2. The other two targets were selected such that either was $3 / 4$ of the way toward one of the distractors (see Figure 8 ).

\section{Method}

Subject, Equipment, and Stimuli. Data are provided from Subject B.B. Stimuli were presented using an Apple Power Macintosh 6100 and Apple high-resolution 13-in. RGB monitor $(640 \times$ 480 pixels). The distractors (D3 and D4) were presented in equal quantities, and set size was fixed at 32 . The colors used were as in Experiment 2 (T, D4, and D3, background) with the addition of $\mathrm{T}^{\prime}$ $\left(u^{\prime}=.197, v^{\prime}=.545\right)$ and $\mathrm{T}^{\prime \prime}\left(u^{\prime}=.217, v^{\prime}=.541\right)$. This configuration is diagrammed schematically in Figure 8. The positioning of $T^{\prime}$ was such that the lengths $D^{\prime} T^{\prime}: T^{\prime} D 3$ are in a $1: 3$ ratio. Similarly, D4T": $T$ " D3 is in a 3:1 ratio.

Table 3

Experiment 3: Mean Reaction times (RT; in Milliseconds) and Percent (\%) Errors for 1:0, 3:1, and 1:1 Conditions

\begin{tabular}{cccrrr} 
& \multicolumn{2}{c}{ Target Absent } & & \multicolumn{2}{c}{ Target Present } \\
\cline { 2 - 3 } \cline { 5 - 6 } Condition & RT & \% Error & & RT & \% Error \\
\hline $1: 0$ & 524 & 2.1 & & 451 & 3.6 \\
$3: 1$ & 1,723 & 0.8 & & 1,025 & 11.5 \\
$1: 1$ & 1,550 & 2.1 & & 906 & 8.2 \\
\hline
\end{tabular}




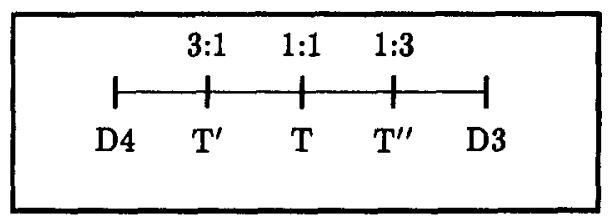

Figure 8. Color configuration for Experiment 3. Colors T, D4, and $D 3$ are the same as in Experiment 2. $T^{\prime}$ and $T^{\prime \prime}$ are at the 3:1 and 1:3 points between Distractors D4 and D3. See text for CIE 1976 UCS coordinates of these equally spaced colors.

Procedure and Analysis. There were five blocks of trials. For two of the blocks, the most extreme color (D4 or D3) was sought in homogeneous displays of the nearest neighboring color ( $\mathrm{T}^{\prime}$ or $\mathrm{T}^{\prime \prime}$, respectively). This was to show that nearest colors are sufficiently distinct, such that if $\mathrm{T}^{\prime}$, for example, were difficult to find in D4 and D3 distractors, one could conclude that it was not because $T^{\prime}$ was indistinguishable from $\mathrm{D} 4$. The three other blocks consisted of search for T', T, or T" in D4 and D3 distractors. Each block contained 138 trials ( 10 practice). The data presented below are based on 1,280 experimental trials (two replications of five blocks of 128 trials). Other trial events were as in Experiments 1 and 2. Outlier screening resulted in rejection of less than $1 \%$ of the observations.

\section{Results and Discussion}

The central question is whether performance improves by moving the target from the $1: 1$ (midway) position to the other two positions $(3: 1$ or $1: 3)$ where the target no longer corresponds to the average of D4 and D3. There is no evidence in the data in Figure 9 that such an improvement occurred. As was the case in Experiment 2 (Figure 7), there appears to have been a small advantage for the 1:1 target. If the additive color hypothesis had been valid, the high point on the graph would have been at the 1:1 point with reduced search times on both sides.

\section{GENERAL DISCUSSION}

The results of three experiments provide an unambiguous answer concerning the additive color hypothesis. In all cases, when the target was not linearly separable from distractors, the target could deviate appreciably from the admix of the distractor chromaticities, and search remained difficult. There is nothing special about placing a target that is not linearly separable precisely at the midpoint. Placing a target that is not linearly separable as extreme as at the 3:1 point (Experiment 3 ) or so that the weighted average of the distractors is quite far from the target (Experiments 1 and 2) did not help search performance. Clearly, linear separability is critical in target conspicuity.

There is another interesting facet to these results that addresses another issue vis-à-vis linear separability as an important determinant of performance in visual search.

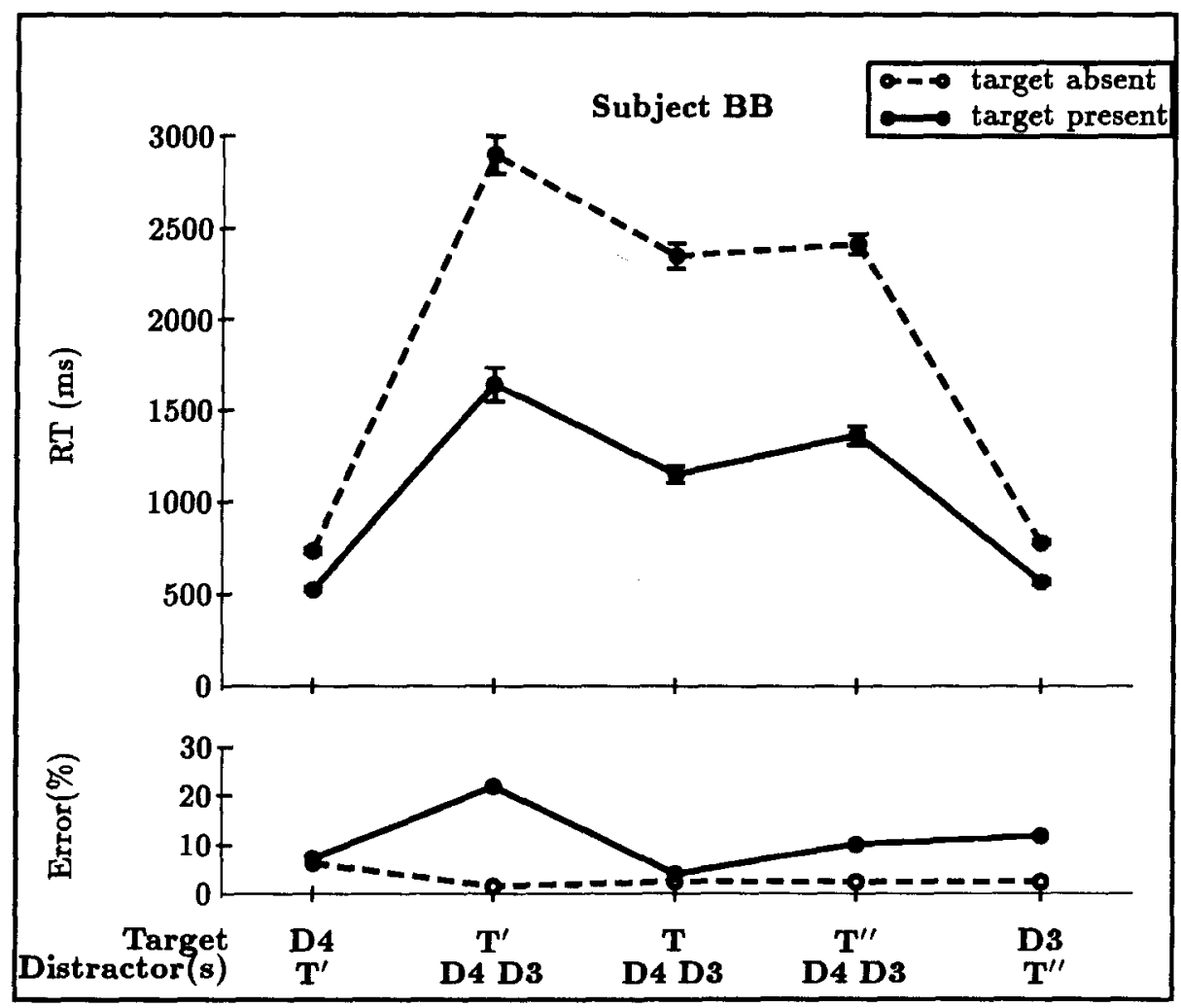

Figure 9. Search results for Subject B.B. in Experiment 3. Error bars ( \pm 1 SEM) may be smaller than the plotting symbol. 
Duncan and Humphreys (1989) and Duncan (1989) argued that there are two factors involved in determining visual search performance: the psychological difference between the target and the distractor(s) (T-D) and the psychological difference between the distractors $(D-D)$. Increased T-D difference leads to easier search, and increased D-D difference leads to more difficult search. For a given pair of distractors, when the target color is midway between these distractors, T-D is minimized, and $\mathrm{D}-\mathrm{D}$ is fixed. When the target moves off the distractordistractor line, T-D increases, and according to the theory proposed by Duncan and Humphreys, and Duncan, slopes should come down. This is precisely what happens. This finding has been recast by Bauer et al. (1996b, 1996c) as an issue of linear separability rather that of increased $\mathrm{T}-\mathrm{D}$ difference.

The results of Experiments 1 and 2 provide additional evidence that T-D and D-D differences are not the sole factors driving the changes in search performance. In the $3: 1$ and $1: 1$ conditions of Experiment $1, T-D$ was fixed, but as the distractor ratio varied, so did the number of $D-D$ differences or heterogeneity. To understand this, consider the $1: 1$ distractor ratio. With both distractors in equal representation, the display will be maximally heterogeneous because many more pairwise comparisons will result in differences than when the ratio becomes increasingly unbalanced. The end condition (1:0) results in homogeneous displays with no heterogeneity. Poisson and Wilkinson (1992) report search data generally consistent with a claim that D-D heterogeneity is largest at 1:1 distractor ratios. In their conjunction searches using orientation and color, the RTs for target-present trials generally peaked near the $1: 1$ ratio, with performance improving as the distractor ratio became increasingly unbalanced. In the 3:1 and 1:1 ratio conditions of Experiment 1, the ratio manipulation had little, if any, effect. What is intriguing is the following: Consider the homogeneous (1:0) condition in which $T$ pops out of D1. In Experiment 1, replacing just three of D1 with D1' (i.e., the 3:1 set-size 16 condition) changed what was essentially set-size-independent search (in the 1:0 condition) into a search where there was a cost of approximately $10 \mathrm{msec} /$ item for target-present trials. Replacing five more D1 with D 1 ' to create a $(1: 1)$ ratio had no pronounced effect on search performance despite an increase in D-D heterogeneity. This is not an explicit test of D-D heterogeneity versus linear separability; however, it does suggest that linear separability per se is a powerful predictor of search performance. This issue is investigated further in Bauer et al. (1996a).

On first consideration, it appears quite surprising that there was no appreciable difference between the 3:1 and $1: 1$ conditions in Experiments 1 and 2 and in the experiments of Bauer et al. (1996a), given what is known about subjects' abilities to search subsets of items to optimize performance (e.g., Egeth, Virzi, \& Garbart 1984; Green \& Anderson, 1956; Kaptein, Theeuwes, \& van der Heijden 1995; Poisson \& Wilkinson 1992; Wolfe, 1992, 1994; Zohary \& Hochstein, 1989). These experiments have demon- strated dramatic performance advantages in adopting the strategy of not searching within a type of distractor that cannot contain the target and searching only the remaining subset of items that could contain the target. For example, in search for a red vertical among red horizontals and green verticals, the green items (or, alternately, the horizontal items) can be safely ignored because the target is red (or, alternately, vertical). That is, a search of all red items only will guarantee detection of the target.

From this perspective, the results of Experiment 2, in which the trials were blocked by ratio, seem especially puzzling. However, in the present experiments, a search within either distractor type (irrespective of numerosity) would not help the subject find the target because the target was never a member of either distractor type. Whether ratios more extreme than those tested in the present experiments would result in enhanced performance is an issue for further experimentation. At this point, we merely wish to dismiss the potential argument that the effects attributed to linear separability by D'Zmura (1991) and Bauer et al. (1996b, 1996c) could be explained by the additive color mixture hypothesis. The interpretation of the present results with respect to this issue is clear. Highly similar performance was seen in conditions in which a target color that was not linearly separable was and was not coincident with the admixture of the distractor colors. This shows that the coincidence of the target chromaticity and the admixture of distractor chromaticities is not responsible for the search phenomena documented in D'Zmura and Bauer et al. (1996b, 1996c).

\section{REFERENCES}

Bauer, B, Jolicoeur, P., \& Cowan, W. B. (1996a). Distractor heterogeneity versus linear separability in colour visual search. Perception, 25, $1281-1293$.

Bauer, B., Jolicoeur, P., \& Cowan, W. B. (1996b). A test of the convex hull hypothesis in visual search for colour targets. Unpublished manuscript, University of Waterloo.

Bauer, B., Jolicoeur, P., \& Cowan, W. B. (1996c). Visual search for colour targets that are or are not linearly-separable from distractors. Vision Research, 36, 1439-1466.

CIE (1978). Recommendations on uniform color scales-color-difference equations, psychometric color terms [Suppl. 2 to CIE Publication No. 15 (E-1.3.1) 1971/(TC-1.3)]. Paris: Bureau Central de la CIE.

DunCAN, J. (1989). Boundary conditions on parallel processing in human vision. Perception, 18, 457-469.

Duncan, J., \& Humphreys, G. W. (1989). Visual search and stimulus similarity. Psychological Review, 96, 433-458.

D'Zmura, M. (1991). Color in visual search. Vision Research, 31, 951-966. Egeth, H. E., Virzi, R. A., \& Garbart, H. (1984). Searching for conjunctively defined targets. Journal of Experimental Psychology: Human Perception \& Performance, 10, 32-39.

GreEN, B., \& ANDERSON, L. (1956). Color coding in a visual search task. Journal of Experimental Psychology, 51, 19-24.

Kaptein, N. A., Theeuwes, J., \& van der Heijden, A. H. C. (1995). Search for a conjunctively defined target can be selectively limited to a color-defined subset of elements. Journal of Experimental Psychology: Human Perception \& Performance, 21, 1053-1069.

Poisson, M. E., \& Wilkinson, F. (1992). Distractor ratio and grouping processes in visual conjunction search. Perception, 21, 21-38.

VAN SElst, M., \& Jolicoeur, P. (1994). A solution to the effect of sample size on outlier elimination. Quarterly Journal of Experimental Psychology, 47A, 631-650. 
Wolfe, J. M. (1992). The parallel guidance of visual attention. Psychological Science, 4, 124-131.

WolfE, J. M. (1994). Guided Search 2.0: A revised model of visual search. Psychonomic Bulletin \& Review, 1, 202-238.

WysZecki, G., \& STILES, W. S. (1982). Color science: Concepts and methods, quantitative data and formulae. New York: Wiley.

ZoHARY, E., \& Hochstein, S. (1989). How serial is serial processing in vision? Perception, 18, 191-200.

\section{NOTES}

1. The authors thank Jeff Hovis of the School of Optometry, University of Waterloo, for performing these tests on all subjects in the experiments reported in this paper.

2. With the set sizes fixed at 16 and 32 , it is not possible to achieve exactly the ratios required on a given trial. For example, for set size 16 target-absent, D1:D1' of 3:1 was realized with 3 of D1 and 12 of D1' on some trials and 4 of $\mathrm{D} 1$ and 11 of $\mathrm{Dl}^{\prime}$ on others. This results in ratios of $4: 1$ and $2.75: 1$, respectively. By including 3 of the 2.75:1 trials for every $4: 1$ trial, the average ratio of $3: 1$ was achieved.

3. The two $1: 1$ ratio conditions produce identical trials, resulting in a potential differential practice effect in the 1:1 condition. This could lead to a reduction in slope for this condition, and, therefore, the lack of slope difference between $3: 1$ and 1:1 would not be surprising. We tested this hypothesis by computing slopes based on only the trials from the first half of the two blocks in the 1:1 condition and including all trials in the 3:1 condition. These results failed to support the differential practice hypothesis; for the 3 subjects, slopes across the two ratio conditions were similar

\begin{tabular}{|c|c|c|}
\hline \multicolumn{3}{|c|}{$\begin{array}{l}\text { APPENDIX } \\
\text { CIE Coordinates of Colors From Figure } 3\end{array}$} \\
\hline Color & $\operatorname{CIE}(x, y)$ & $\operatorname{CIE}\left(u^{\prime}, v^{\prime}\right)$ \\
\hline \multicolumn{3}{|c|}{ Subject B.G. } \\
\hline D1 & $.441, .423$ & $.245, .529$ \\
\hline D2 & $.434, .399$ & $.251, .519$ \\
\hline $\mathrm{T}$ & $.427, .375$ & $.257, .508$ \\
\hline $\mathrm{D} 1^{\prime}$ & $.422, .355$ & $.263, .498$ \\
\hline $\mathrm{D} 2{ }^{\prime}$ & $.415, .334$ & $.269, .487$ \\
\hline \multicolumn{3}{|c|}{ Subject T.O. } \\
\hline D1 & $.454, .415$ & $.257, .528$ \\
\hline D2 & $.440, .394$ & $.257, .518$ \\
\hline $\mathrm{T}$ & $.427, .375$ & $.257, .508$ \\
\hline $\mathrm{D} 1^{\prime}$ & $.415, .357$ & $.257, .498$ \\
\hline $\mathrm{D} 2^{\prime}$ & $.403, .340$ & $.257, .488$ \\
\hline \multicolumn{3}{|c|}{ Subject B.B. } \\
\hline D1 & $.417, .409$ & $.236, .520$ \\
\hline D2 & $.423, .393$ & $.246, .515$ \\
\hline $\mathrm{T}$ & $.427, .375$ & $.257, .508$ \\
\hline $\mathrm{D} 1^{\prime}$ & $.432, .361$ & $.267, .502$ \\
\hline $\mathrm{D} 2^{\prime}$ & $.436, .346$ & $.278, .496$ \\
\hline Background & $.287, .292$ & $.194, .443$ \\
\hline
\end{tabular}

(Manuscript received May 21, 1996; revision accepted for publication August 5, 1997.) 